SLAC-PUB-7450

WIS-97/10/Apr-PH

hep-ph/9704287

\title{
A Model Independent Construction of the Unitarity Triangle
}

\author{
Yuval Grossman ${ }^{a}$, Yosef Nir ${ }^{b}$ and Mihir P. Worah ${ }^{a}$ \\ ${ }^{a}$ Stanford Linear Accelerator Center, Stanford University, Stanford, CA 94309, USA \\ ${ }^{b}$ Department of Particle Physics, Weizmann Institute of Science, Rehovot 76100, Israel
}

In a large class of models, the only significant new physics effect on the $\mathrm{CP}$ asymmetries in $B \rightarrow \psi K_{S}$ and $B \rightarrow \pi \pi$ decays is a new contribution to the $B-\bar{B}$ mixing amplitude. This allows a model independent construction of the CKM Unitarity Triangle (up to hadronic uncertainties). Furthermore, the contributions to the mixing from the Standard Model and from the new physics can be disentangled. A serious obstacle to this analysis is an eightfold discrete ambiguity in solving for the angles of the triangle. Several ways to reduce the ambiguity either by making further measurements, or by making further assumptions about the nature of the new physics are described. 


\section{The Basic Assumptions and Results}

The first two CP asymmetries to be measured in a $B$ factory are likely to be [1]

$$
\begin{gathered}
\frac{\Gamma\left(B_{\text {phys }}^{0}(t) \rightarrow \psi K_{S}\right)-\Gamma\left(\bar{B}_{\text {phys }}^{0}(t) \rightarrow \psi K_{S}\right)}{\Gamma\left(B_{\text {phys }}^{0}(t) \rightarrow \psi K_{S}\right)+\Gamma\left(\bar{B}_{\text {phys }}^{0}(t) \rightarrow \psi K_{S}\right)}=-a_{\psi K_{S}} \sin \left(\Delta m_{B} t\right), \\
\frac{\Gamma\left(B_{\text {phys }}^{0}(t) \rightarrow \pi \pi\right)-\Gamma\left(\bar{B}_{\text {phys }}^{0}(t) \rightarrow \pi \pi\right)}{\Gamma\left(B_{\text {phys }}^{0}(t) \rightarrow \pi \pi\right)+\Gamma\left(\bar{B}_{\text {phys }}^{0}(t) \rightarrow \pi \pi\right)}=-a_{\pi \pi} \sin \left(\Delta m_{B} t\right) .
\end{gathered}
$$

In addition, the $B$ factory will improve our knowledge of the $B-\bar{B}$ mixing parameter, $x_{d} \equiv \frac{\Delta m_{B}}{\Gamma_{B}}$, and of the charmless semileptonic branching ratio of the $B$ mesons.

Within the Standard Model, these four measurements are useful in constraining the unitarity triangle. The asymmetries (1.1) and (1.2) measure angles of the unitarity triangle:

$$
\begin{gathered}
a_{\psi K_{S}}=\sin 2 \beta, \\
a_{\pi \pi}=\sin 2 \alpha,
\end{gathered}
$$

where

$$
\alpha \equiv \arg \left[-\frac{V_{t d} V_{t b}^{*}}{V_{u d} V_{u b}^{*}}\right], \quad \beta \equiv \arg \left[-\frac{V_{c d} V_{c b}^{*}}{V_{t d} V_{t b}^{*}}\right] .
$$

In (1.3) we have taken into account the fact that the final state is CP-odd. In (1.4) we have ignored possible penguin contamination which can, in principle, be eliminated by isospin analysis [2]. The measurement of $x_{d}$ determines one side of the unitarity triangle $\left(R_{t}\right)$ :

$$
x_{d}=C_{t} R_{t}^{2},
$$

where

$$
R_{t} \equiv\left|\frac{V_{t b}^{*} V_{t d}}{V_{c b}^{*} V_{c d}}\right|
$$

and $C_{t}=\tau_{b} \frac{G_{F}^{2}}{6 \pi^{2}} \eta_{B} m_{B}\left(B_{B} f_{B}^{2}\right) m_{t}^{2} f_{2}\left(m_{t}^{2} / m_{W}^{2}\right)\left|V_{c b}^{*} V_{c d}\right|^{2}$ (for definitions and notations see [1]). The present values are $x_{d}=0.73 \pm 0.05$ and $C_{t} \sim 0.4-0.8$ for $\sqrt{B_{B}} f_{B}=140-200$ $\mathrm{MeV}$ [3]. Measurements of various inclusive and exclusive $b \rightarrow u \ell \nu$ processes will determine (up to uncertainties arising from various hadronic models) the length of the other side of the unitarity triangle $\left(R_{u}\right)$ :

$$
\frac{\Gamma(b \rightarrow u \ell \nu)}{\Gamma(b \rightarrow c \ell \nu)}=\frac{1}{F_{\mathrm{ps}}}\left|\frac{V_{c d}}{V_{u d}}\right|^{2} R_{u}^{2}
$$


where

$$
R_{u} \equiv\left|\frac{V_{u b}^{*} V_{u d}}{V_{c b}^{*} V_{c d}}\right|
$$

and $F_{\mathrm{ps}} \approx 0.5$ is a phase space factor. The present value for $R_{u}$ ranges from 0.27 to 0.45 depending on the hadronic model used to relate the measurement at the endpoint region, or of some exclusive mode, to the total $b \rightarrow u$ inclusive rate [3].

In the presence of new physics it is quite possible that the Standard Model predictions (1.3), (1.4) and (1.6) are violated. The most likely reason is a new, significant contribution to $B-\bar{B}$ mixing that carries a $\mathrm{CP}$ violating phase different from the Standard Model one. Other factors that could affect the construction of the unitarity triangle from these four measurements are unlikely to be significant [4뎨:

a. The $\bar{b} \rightarrow \bar{c} c \bar{s}$ and $\bar{b} \rightarrow \bar{u} u \bar{d}$ decays for $a_{\psi K_{S}}$ and $a_{\pi \pi}$ respectively, as well as the semileptonic $B$ decays for $R_{u}$, are mediated by Standard Model tree level diagrams. In most extensions of the Standard Model there is no decay mechanism that could significantly compete with these contributions. (For exceptions, which could affect the $\bar{b} \rightarrow \bar{u} u \bar{d}$ decay, see [6].)

b. New physics could contribute significantly to $K-\bar{K}$ mixing. However, the small value of $\varepsilon_{K}$ forbids large deviations from the Standard Model phase of the mixing amplitude.

c. Unitarity of the three generation CKM matrix is maintained if there are no quarks beyond the three generations of the Standard Model. Even in models with an extended quark sector the effect on $B-\bar{B}$ mixing is always larger than the violation of CKM unitarity.

Our analysis below applies to models where the above three conditions are not significantly violated. Under these circumstances the relevant new physics effects can be described by two new parameters, $r_{d}$ and $\theta_{d}$ [77-10], defined by

$$
\left(r_{d} e^{i \theta_{d}}\right)^{2} \equiv \frac{\left\langle B^{0}\left|\mathcal{H}_{\mathrm{eff}}^{\text {full }}\right| \bar{B}^{0}\right\rangle}{\left\langle B^{0}\left|\mathcal{H}_{\mathrm{eff}}^{\mathrm{SM}}\right| \bar{B}^{0}\right\rangle},
$$

where $\mathcal{H}_{\text {eff }}^{\text {full }}$ is the effective Hamiltonian including both Standard Model and new physics contributions, and $\mathcal{H}_{\mathrm{eff}}^{\mathrm{SM}}$ only includes the Standard Model box diagrams. In particular, 
with this definition, the modification of the two $\mathrm{CP}$ asymmetries in (1.3) and (1.4) depends on a single new parameter, the phase $\theta_{d}$ :

$$
\begin{gathered}
a_{\psi K_{S}}=\sin \left(2 \beta+2 \theta_{d}\right), \\
a_{\pi \pi}=\sin \left(2 \alpha-2 \theta_{d}\right),
\end{gathered}
$$

while the modification of the $B-\bar{B}$ mixing parameter $x_{d}$ in (1.6) is given by the magnitude rescaling parameter, $r_{d}$ :

$$
x_{d}=C_{t} R_{t}^{2} r_{d}^{2} .
$$

Furthermore, since the determination of $R_{u}$ from the semileptonic $B$ decays is not affected by the new physics, and since the unitarity triangle remains valid, we have the following relations between the length of its sides and its angles:

$$
\begin{aligned}
R_{u} & =\frac{\sin \beta}{\sin \alpha}, \\
R_{t} & =\frac{\sin \gamma}{\sin \alpha},
\end{aligned}
$$

where

$$
\gamma \equiv \arg \left[-\frac{V_{u d} V_{u b}^{*}}{V_{c d} V_{c b}^{*}}\right] .
$$

When $\alpha, \beta$ and $\gamma$ are defined to lie in the $\{0,2 \pi\}$ range, they satisfy

$$
\alpha+\beta+\gamma=\pi \text { or } 5 \pi
$$

The four measured quantities $a_{\psi K_{S}}, a_{\pi \pi}, x_{d}$ and $R_{u}$ can be used to achieve the following [7]:

(i) Fully reconstruct the unitarity triangle and, in particular, find $\alpha, \beta$ and $R_{t}$;

(ii) Find the magnitude and phase of the new physics contribution to $B-\bar{B}$ mixing, namely determine $r_{d}$ and $\theta_{d}$.

It is straightforward to show that the above tasks are possible. Eqs. (1.11), (1.12) and (1.14) give three equations for three unknowns, $\alpha, \beta$ and $\theta_{d}$. Once $\alpha$ and $\beta$ are known, $\gamma$ can be extracted from (1.17), $R_{t}$ can then be deduced from (1.15), and finally $r_{d}$ is found from (1.13). 
In practice, however, it is quite likely that the combination of experimental and theoretical uncertainties (particularly in the $x_{d}$ and $R_{u}$ constraints) and discrete ambiguities will limit the usefulness of the above method rather significantly. In the next section we discuss the discrete ambiguities that arise in this calculation. We then describe how to determine the parameters, both in the $\rho-\eta$ plane (section 3 ), and in the $\sin 2 \alpha-\sin 2 \beta$ plane (section 4). We mention ways to resolve some of the ambiguities in the concluding section.

\section{Discrete Ambiguities}

A major obstacle in carrying out the above program will be the discrete ambiguities in determining $\gamma$. We now describe these ambiguities.

A physically meaningful range for an angle is $2 \pi$. We choose this range to be $\{0,2 \pi\}$. Measurement of any single asymmetry, $\sin 2 \phi$, determines the corresponding angle only up to a fourfold ambiguity: $\phi, \pi / 2-\phi, \pi+\phi$ and $3 \pi / 2-\phi(\bmod 2 \pi)$. Specifically, let us denote by $\bar{\alpha}$ and $\bar{\beta}$ some solution of the equations

$$
a_{\psi K_{S}}=\sin 2 \bar{\beta}, \quad a_{\pi \pi}=\sin 2 \bar{\alpha}
$$

Thus, measurements of the two asymmetries leads to a sixteenfold ambiguity in the values

of the $\{\bar{\alpha}, \bar{\beta}\}$ pair. However, since $\bar{\alpha}=\alpha-\theta_{d}$ and $\bar{\beta}=\beta+\theta_{d}$, and unitarity is not violated, $\gamma$ still satisfies the condition

$$
\bar{\alpha}+\bar{\beta}+\gamma=\pi(\bmod 2 \pi)
$$

Then, the sixteen possibilities for $\gamma$ are divided into two groups of eight that are related by the combined operation $\bar{\alpha} \rightarrow \bar{\alpha}+\pi$ and $\bar{\beta} \rightarrow \bar{\beta}+\pi$. This, in turn shifts the value of $\gamma$ by $2 \pi$. However, since $\gamma$ is only defined modulo $2 \pi$, the ambiguity in $\gamma$ is reduced to eightfold. We emphasize that this reduction of the ambiguity depends only on the definition of $\gamma$. Defining

$$
\phi_{ \pm}=\bar{\alpha} \pm \bar{\beta}
$$


the eight possible solutions for $\gamma$ are

$$
\gamma= \pm \phi_{+}, \pi \pm \phi_{+}, \pi / 2 \pm \phi_{-}, 3 \pi / 2 \pm \phi_{-}(\bmod 2 \pi)
$$

Note that the eight solutions come in pairs of $\pm \gamma$. This in turn implies that the ambiguity on $R_{t}$ is only fourfold.

In any model where the three angles $\bar{\alpha}, \bar{\beta}$, and $\gamma$ form a triangle, the ambiguity is further reduced [11]: the requirement that the angles are either all in the range $\{0, \pi\}$ or all in the range $\{\pi, 2 \pi\}$ reduces the ambiguity in $\gamma$ to fourfold. It is enough to know the signs of $a_{\psi K_{S}}$ and $a_{\pi \pi}$ to carry out this step. Finally, within the Standard Model, the bound $0<\beta<\pi / 4$ (obtained from the sign of $\varepsilon_{K}$ and from $R_{u}<1 / \sqrt{2}$ ) reduces the ambiguity in $\gamma$ to twofold.

When we allow for the possibility of new physics effects in the mixing, knowing the signs of $a_{\psi K_{S}}$ and $a_{\pi \pi}$ does not lead to further reduction in the ambiguity, which remains eightfold. The three angles $\bar{\alpha}, \bar{\beta}$ and $\gamma$ are not angles that define a triangle and therefore further constraints cannot be imposed. It is possible, for example, that both $\gamma$ and $\bar{\beta}$ lie in the range $\{\pi / 2, \pi\}$. Further the sign of $\varepsilon_{K}$ may not be related to the sign of $\eta$.

The following example will make the situation clear. Take

$$
a_{\pi \pi}=1 / 2, \quad a_{\psi K_{S}}=\sqrt{3} / 2
$$

Then, we could have

$$
\bar{\alpha}=\frac{\pi}{12}, \frac{5 \pi}{12}, \frac{13 \pi}{12}, \frac{17 \pi}{12}, \quad \bar{\beta}=\frac{\pi}{6}, \frac{\pi}{3}, \frac{7 \pi}{6}, \frac{4 \pi}{3} .
$$

The eight solutions for $\gamma$ are

$$
\gamma=\frac{\pi}{4}, \frac{5 \pi}{12}, \frac{7 \pi}{12}, \frac{3 \pi}{4}, \frac{5 \pi}{4}, \frac{17 \pi}{12}, \frac{19 \pi}{12}, \frac{7 \pi}{4}
$$

If $\bar{\alpha}, \bar{\beta}, \gamma$ define a triangle, then only four solutions are allowed:

$$
(\bar{\alpha}, \bar{\beta}, \gamma)=\left(\frac{\pi}{12}, \frac{\pi}{6}, \frac{3 \pi}{4}\right),\left(\frac{\pi}{12}, \frac{\pi}{3}, \frac{7 \pi}{12}\right),\left(\frac{5 \pi}{12}, \frac{\pi}{6}, \frac{5 \pi}{12}\right),\left(\frac{5 \pi}{12}, \frac{\pi}{3}, \frac{\pi}{4}\right) .
$$

Assuming $0<\bar{\beta}<\pi / 4$ as in the Standard Model leaves only the first two choices. 
In various specific cases, the discrete ambiguity is smaller. If the two asymmetries are equal in magnitude, there is only a sixfold ambiguity:

$$
\begin{gathered}
a_{\pi \pi}=a_{\psi K_{S}} \Longrightarrow \gamma= \pm 2 \bar{\beta}, \pi \pm 2 \bar{\beta}, \pi / 2,3 \pi / 2(\bmod 2 \pi), \\
a_{\pi \pi}=-a_{\psi K_{S}} \Longrightarrow \gamma=0, \pi, \pi / 2 \pm 2 \bar{\beta}, 3 \pi / 2 \pm 2 \bar{\beta}(\bmod 2 \pi) .
\end{gathered}
$$

If one of the asymmetries is maximal, there is a fourfold ambiguity, e.g.

$$
\begin{array}{lll}
a_{\pi \pi}=+1 \Longrightarrow \gamma= \pm(\pi / 4+\bar{\beta}), & \pm(3 \pi / 4-\bar{\beta}) & (\bmod 2 \pi), \\
a_{\pi \pi}=-1 \Longrightarrow \gamma= \pm(\pi / 4-\bar{\beta}), & \pm(3 \pi / 4+\bar{\beta}) & (\bmod 2 \pi) .
\end{array}
$$

If both asymmetries are maximal, the ambiguity is twofold. If the two asymmetries vanish, there is only a fourfold ambiguity:

$$
a_{\pi \pi}=a_{\psi K_{S}}=0 \Longrightarrow \gamma=0, \pi / 2, \pi, 3 \pi / 2
$$

This is an interesting case, because it is predicted by models with approximate CP symmetry (e.g. in some supersymmetric models [12]). Only two of the solutions $(0, \pi)$ correspond to the $\mathrm{CP}$ symmetric case while in the other two $(\pi / 2,3 \pi / 2)$, the zero asymmetries are accidental.

So far we have ignored the penguin contamination in $a_{\pi \pi}$. The isospin analysis eliminates the penguin contamination only up to a four fold ambiguity [2]. Therefore, if the isospin analysis is needed, the ambiguities are increased.

In addition, for each value of $\gamma$ there are two possibilities for $\theta_{d}$ related by $\theta_{d} \rightarrow \theta_{d}+\pi$. As long as the new physics is such that the $\Delta b=2$ operator that contributes to $B-\bar{B}$ mixing can be separated into two $\Delta b=1$ operators the $\theta_{d} \rightarrow \theta_{d}+\pi$ ambiguity is physical. Otherwise, it is not physical.

\section{The $\rho-\eta$ Plane}

The key point in the extraction of the CKM parameters is that the angle $\theta_{d}$ cancels in the following sum:

$$
2(\alpha+\beta)=\arcsin \left(a_{\psi K_{S}}\right)+\arcsin \left(a_{\pi \pi}\right) .
$$


In other words, the angle $\gamma$ can be determined (up to the discrete ambiguities discussed above). In the $\rho-\eta$ plane, a value for $\gamma$ gives a ray from the origin, while a value for $R_{u}$ gives a circle that is centered in the origin. The intersection point of the line and the circle gives $(\rho, \eta)$ of the unitarity triangle and determines it completely.

A graphical way to carry out these calculations in the $\rho-\eta$ plane is the following (see Figure 1) [10]. One draws the four curves that correspond to eqs. (1.3), (1.4), (1.6) and (1.8) (even though only the latter is valid!). The next step is to draw the ray from the origin that passes through the intersection point of the $\beta$-ray and the $\alpha$-circle: this is the correct $\gamma$-ray (see the dashed line in Figure 1). The intersection point of the $\gamma$-ray and the $R_{u}$-circle gives the correct vertex of the unitarity triangle, $(\rho, \eta)$, namely

$$
\begin{aligned}
\tan \beta & =\frac{\eta}{1-\rho}, \\
R_{t}^{2} & =\eta^{2}+(1-\rho)^{2} .
\end{aligned}
$$

The information about the new physics contribution to $B-\bar{B}$ mixing is found from the intersection point of the $\beta$-ray and the $x_{d^{-}}$circle, $\left(\rho^{\prime}, \eta^{\prime}\right)$, namely

$$
\begin{aligned}
\theta_{d} & =\arctan \frac{\eta^{\prime}}{1-\rho^{\prime}}-\arctan \frac{\eta}{1-\rho} \\
r_{d}^{2} & =\frac{\eta^{2}+\left(1-\rho^{\prime}\right)^{2}}{\eta^{2}+(1-\rho)^{2}}
\end{aligned}
$$

\section{The $\sin 2 \alpha-\sin 2 \beta$ Plane}

A presentation of the various constraints in the $\sin 2 \alpha-\sin 2 \beta$ plane [7, 13, 14 is useful because the two angles are usually correlated [15]. The model independent analysis is demonstrated in Figure 2. The $R_{u}$ constraint gives an eight-shaped curve on which the physical values have to lie. The various solutions for eq. (3.1) fall on two ellipses, the intersections of which with the $R_{u}$ curve determine the allowed values of $\sin 2 \alpha$ and $\sin 2 \beta$. Note that these ellipses cross the eight-shaped curve in sixteen points but, as argued above, only eight of these points are true solutions. The inconsistent intersection points can be found by noting that the slopes of the ellipse at the consistent points should be $(\cos 2 \alpha,-\cos 2 \beta)$. The eight correct solutions are denoted by the filled circles in Figure 2 . 
In the above, we showed how to use measured values of the $\mathrm{CP}$ asymmetries $a_{\psi K_{S}}$ and $a_{\pi \pi}$ to find the allowed values for $\alpha$ and $\beta$. The presentation in the $\sin 2 \alpha-\sin 2 \beta$ plane is also useful for the opposite situation. Some models predict specific values for $\alpha$ and $\beta$. (Such predictions can arise naturally from horizontal symmetries.) On the other hand, the models often allow new contributions to $B-\bar{B}$ mixing of unknown magnitude and phase. In this case, the predicted value of $(\sin 2 \alpha, \sin 2 \beta)$ is just a point in the plane, and the ellipse (3.1) actually gives the allowed (and correlated) values of $\left(a_{\pi \pi}, a_{\psi K_{S}}\right)$. Such an analysis was carried out in ref. [16].

More generally, even in models that make no specific predictions for CKM parameters, we usually have some constraints on the allowed range for $\alpha$ and $\beta$. For example, in this work we assume the validity of the limits on $R_{u}$ from charmless semileptonic $B$ decays which constrains the ratio $\sin \beta / \sin \alpha$ through (1.14). Note, however, that this constraint by itself cannot exclude any region in the $a_{\pi \pi}-a_{\psi K_{S}}$ plane. The reason is the following. For any value of $R_{u}$, neither $\alpha$ nor $\theta_{d}$ are constrained. (The angle $\beta$ is constrained for any $R_{u}<1$ and certainly by the present range, $0.27<R_{u}<0.45$.) Then any value of $a_{\psi K_{S}}$ can be accommodated by an appropriate choice of $\theta_{d}$ and any value of $a_{\pi \pi}$ can be fitted by further choosing an appropriate $\alpha$. Obviously, to get predictions for the CP asymmetries beyond the Standard Model, one has to make some assumptions that go beyond our generic analysis.

For example, consider models where $\varepsilon_{K}$ is dominated by the Standard Model box diagrams (while $B-\bar{B}$ mixing is not). Then, we know that $0<\gamma<\pi$. This already excludes part of the allowed range. In particular, $\left(a_{\pi \pi}, a_{\psi K_{S}}\right)=(1,-1)$ or $(-1,1)$ requires $\gamma=0$ or $\pi$, and is therefore excluded in this class of models. More generally, in any class

of models where $\sin ^{2} \gamma$ cannot assume any value between zero and one, some regions in the $a_{\pi \pi}-a_{\psi K_{S}}$ plane are excluded.

\section{Final Comments}

We argued that the most likely effect of new physics on CP asymmetries in neutral $B$ decays into CP eigenstates will be a significant contribution to the mixing. This is because 
we have concentrated on decays that are allowed at tree level in the Standard Model. Thus the new physics effects on the decay amplitudes and on CKM unitarity can be neglected in a large class of models 1 . We explained that in this class of models, the unitarity triangle can be constructed model independently and the new physics contribution to the mixing can be disentangled from the Standard Model one.

However, the combination of hadronic uncertainties and discrete ambiguities puts serious obstacles in carrying out this calculation. In particular, there is an eightfold ambiguity in the construction of the triangle. In order to get useful results, it will be necessary to reduce this ambiguity.

One way to eliminate some of the allowed solutions can be provided by a rough knowledge of $\cos \left(2 \alpha-2 \theta_{d}\right), \cos \left(2 \beta+2 \theta_{d}\right)$ or $\cos 2 \gamma$ [17]. For example, $\cos \left(2 \alpha-2 \theta_{d}\right)$ can be determined from the CP asymmetry in $B \rightarrow \rho \pi$ [18] and $\cos 2 \gamma$ from $B \rightarrow D K$ [19]. While a precise measurement of either of these is not expected in the first stages of a $B$ factory, a knowledge of the sign of the cosine is already useful for our purposes: knowing either of $\operatorname{sign}\left[\cos 2\left(\alpha-\theta_{d}\right)\right], \operatorname{sign}\left[\cos 2\left(\beta+\theta_{d}\right)\right]$ or $\operatorname{sign}[\cos 2 \gamma]$ reduces the ambiguity in $\gamma$ to fourfold. Knowing two of them reduces it to twofold. (Knowing the three of them, however, cannot be combined to completely eliminate the ambiguity.)

The ambiguity associated with the isospin analysis can be removed by measuring the time dependent $\mathrm{CP}$ asymmetry in $B \rightarrow \pi^{0} \pi^{0}$ [2]. Another way is by studying $B \rightarrow \rho \pi$ [18,17]. Here, due to interference between several amplitudes, isospin relations can be used to determine $\sin 2 \alpha$ without penguin contamination, and without any discrete ambiguity.

A different approach is to make further assumptions about the new physics that is responsible for the effects discussed above. For example, there are many models where processes involving third generation quarks, such as $B-\bar{B}$ mixing, are significantly modified by the new physics, but processes with only light quarks, such as $K \rightarrow \pi \nu \bar{\nu}$, are not. Then measurements of $K^{+} \rightarrow \pi^{+} \nu \bar{\nu}$ and $K_{L} \rightarrow \pi^{0} \nu \bar{\nu}$ will provide the true values of $R_{t}$ or $|\eta|$, respectively. The unitarity triangle can be determined from these up to a fourfold ambiguity. The additional input of $R_{u}$ reduces this to a twofold ambiguity. The

1 The new physics effects may significantly alter the patterns of CP asymmetries in decays that are dominated by penguins in the Standard Model [6]. 
determination of $\gamma$ by the methods described above will provide a test of this class of models. It will not resolve the twofold ambiguity.

In some models [20] there is a significant contribution to both $B_{d}$ and $B_{s}$ mixing but the ratio between the two obeys the Standard Model relation,

$$
\frac{\Delta m_{B_{d}}}{\Delta m_{B_{s}}}=F_{S U(3)} \sin ^{2} \theta_{C} R_{t}^{2}
$$

where $F_{S U(3)}$ is an $S U(3)$-isospin breaking parameter. Then, a measurement of $\Delta m_{B_{s}}$ will provide the correct $R_{t}$ and, again, the unitarity triangle can be determined, up to a twofold discrete ambiguity, from $R_{u}$ and $R_{t}$. The determination of $\gamma$ by our analysis is in this case, again, a test and will not resolve the twofold ambiguity. Note, however, that in most models where the ratio between $B_{d}$ and $B_{s}$ mixing obeys (5.1), the phases in the $B_{s}, B_{d}$ mixing amplitudes are the same as in the Standard Model, namely $\theta_{d}=0$. Then $r_{d}$ is the only new parameter, and the whole analysis becomes trivial.

In a large class of models, $\varepsilon_{K}$ has only small contributions from new physics. If dominated by the Standard Model, $\varepsilon_{K}$ implies that all angles of the unitarity triangle are in the range $\{0, \pi\}$, and the ambiguity is reduced to fourfold.

Of course, one can combine several of these measurements and assumptions to get a better handle on the true form of the unitarity triangle. It is obvious however that the model independent construction of the triangle, while possible in principle, will pose a serious theoretical and experimental challenge.

\section{Acknowledgments}

We thank Ronen Plesser and Helen Quinn for useful discussions. Y.G. and M.P.W. are supported by the Department of Energy under contract DE-AC03-76SF00515. Y.N. is supported in part by the United States - Israel Binational Science Foundation (BSF), by the Israel Science Foundation, and by the Minerva Foundation (Munich). 


\section{References}

[1] For a review see Y. Nir and H.R. Quinn, Ann. Rev. Nucl. Part. Sci. 42 (1992) 211.

[2] M. Gronau and D. London, Phys. Rev. Lett. 65 (1990) 3381.

[3] R.M. Barnett et al. [Particle Data Group], Phys. Rev. D54 (1996) 1.

[4] Y. Nir and D. Silverman, Nucl. Phys. B345 (1990) 301.

[5] C.O. Dib, D. London and Y. Nir, Int. J. Mod. Phys. A6 (1991) 1253.

[6] Y. Grossman and M. Worah, Phys. Lett. B395 (1997) 241.

[7] J.M. Soares and L. Wolfenstein, Phys. Rev. D47 (1993) 1021.

[8] N.G. Deshpande, B. Dutta and S. Oh, Phys. Rev. Lett. 77 (1996) 4499.

[9] J.P. Silva and L. Wolfenstein, hep-ph/9610208.

[10] A.G. Cohen, D.B. Kaplan, F. Lepeintre and A.E. Nelson, Phys. Rev. Lett. 78 (1997) 2300.

[11] Y. Nir and H.R. Quinn, Phys. Rev. D42 (1990) 1473.

[12] For a review, see Y. Grossman, Y. Nir and R. Rattazzi, hep-ph/9701231.

[13] Y. Nir and U. Sarid, Phys. Rev. D47 (1993) 2818.

[14] Y. Grossman and Y. Nir, Phys. Lett. B313 (1993) 126.

[15] C.O. Dib, I. Dunietz, F.J. Gilman and Y. Nir, Phys. Rev. D41 (1990) 1522.

[16] R. Barbieri, L.J. Hall and A. Romanino, hep-ph/9702315.

[17] Y. Grossman and H.R. Quinn, SLAC-PUB-7454, in preparation.

[18] A.E. Snyder and H.R. Quinn, Phys. Rev. D48 (1993) 2139.

[19] M. Gronau and D. Wyler, Phys. Lett. B265 (1991) 172.

[20] Y. Nir, Phys. Lett. B327 (1994) 85. 


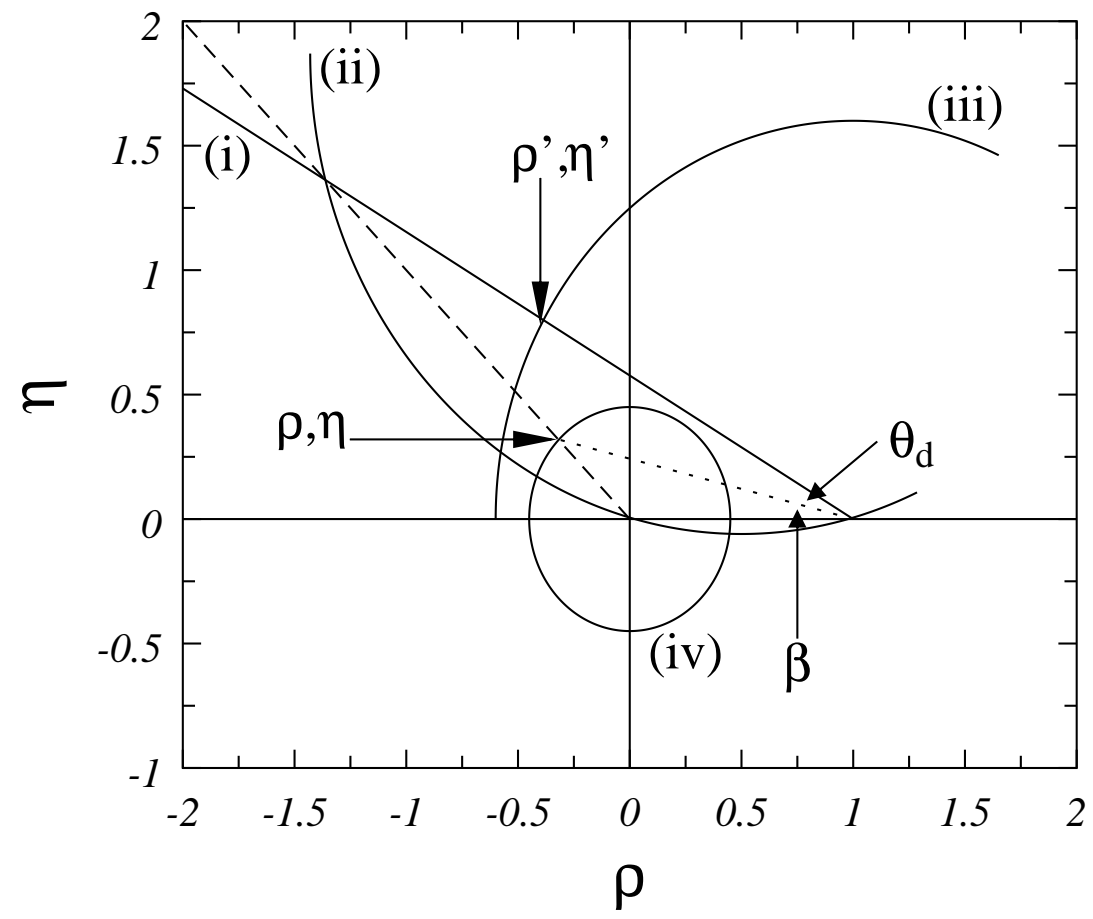

Figure 1. The model independent analysis in the $\rho-\eta$ plane: (i) The $a_{\psi K_{S}}$ ray; (ii) The $a_{\pi \pi}$ circle; (iii) The $x_{d}$ circle; (iv) The $R_{u}$ circle. The $\gamma$ ray is given by the dashed line. The true $\beta$ ray is given by the dotted line. Also shown are the true vertex of the unitarity triangle $(\rho, \eta)$ and the $\left(\rho^{\prime}, \eta^{\prime}\right)$ point that serves to find $\theta_{d}$ and $r_{d}$. 


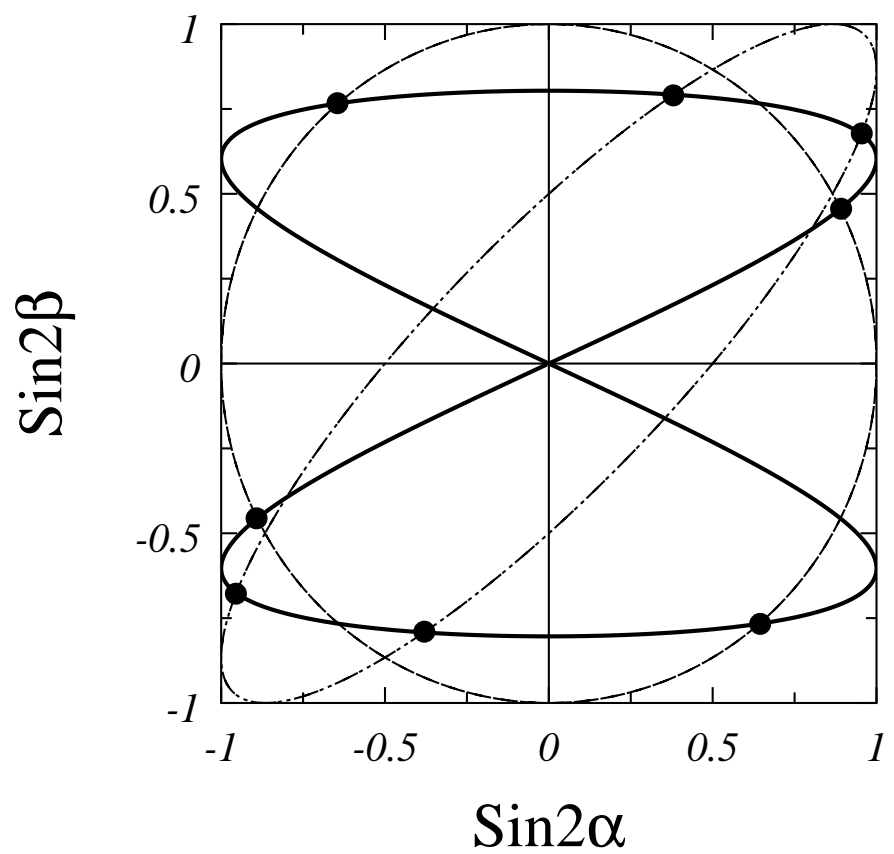

Figure 2. The $\alpha+\beta$ constraint (3.1) and the $R_{u}$ constraint (1.14) in the $\sin 2 \alpha-\sin 2 \beta$ plane. The eight possible solutions for the unitarity triangle are given by the filled circles. 